\title{
LOS DOCENTES QUE CREEN EN LA INCLUSIÓN EDUCATIVA ¿TIENEN UNA ACTITUD FAVORABLE HACIA EL ALUMNADO DE ALTAS CAPACIDADES?
}

\author{
María del Carmen Plata Morán \\ Facultad de Formación del profesorado \\ Universidad de Extremadura \\ maplatam@alumnos.unex.es \\ Margarita Gozalo Delgado \\ Facultad de Formación del profesorado \\ Universidad de Extremadura \\ mgozalo@unex.es \\ Carmen Gómez Labrador \\ Consejería de Educación y empleo \\ Junta de Extremadura \\ carmengomezlab@gmail.com. \\ Sabina Barrios Fernández. \\ Facultad de Enfermería y Terapia Ocupacional \\ Universidad de Extremadura \\ sabinabarrios@unex.es
}

Recepción Artículo: 17 mayo 2021 Admisión Evaluación: 17 mayo 2021 Informe Evaluador 1: 19 mayo 2021 Informe Evaluador 2: 26 mayo 2021 Aprobación Publicación: 01 junio 2021

\section{RESUMEN}

Apostar por la inclusión educativa supone promover una educación de calidad para todo el alumnado. Las creencias y actitudes del profesorado hacia la inclusión constituyen un factor que puede influir sobre la calidad de la respuesta educativa que reciben los alumnos. En el caso de las Altas Capacidades, esta relación se ha visto ampliamente probada en estudios nacionales e internacionales, incluso se observa que el profesorado más comprometido con el concepto de inclusión no siempre tiene actitudes favorables hacia este alumnado (McCoach y Siegle, 2007; Tirri, Tallent-Runnels, Adams, Yuen y Lau (2002). El objetivo de este estudio ha sido comprobar qué relación existe entre las actitudes del profesorado hacia la inclusión educativa y hacia los alumnos/as con altas capacidades y su educación. Participaron 203 profesores/as en activo (56 hombres y 147 mujeres) de distintos niveles educativos, con edades entre los 22 y los 74 años, 48.53 (15.5) años, los cuales respondieron dos cuestionarios: Cuestionario de percepciones del profesorado acerca de la inclusión (Cardona, Gómez-Canet y González-Sánchez, 2000) y Opiniones sobre los alumnos con altas capacidades y su educación (Original de Gagné \& Nadeau (1991, adaptado por Tourón, Fernández y Reyero, 2002). Se comprobó que los docentes de 


\title{
LOS DOCENTES QUE CREEN EN LA INCLUSIÓN EDUCATIVA ¿TIENEN UNA ACTITUD FAVORABLE HACIA EL ALUMNADO DE ALTAS CAPACIDADES?
}

menor edad y los mejor formados muestran actitudes más favorables hacia la inclusión, sin embargo, estas características no siempre coinciden cuando valoramos sus actitudes hacia el alumnado de altas capacidades.

Palabras Clave: actitudes profesorado; inclusión educativa; necesidades educativas; alumnos de altas capacidades

\begin{abstract}
Do teachers who believe in educational inclusion have a favorable attitude towards students with gifted?. A commitment to inclusive education means promoting quality education for all learners. Teachers' beliefs and attitudes towards inclusion are a factor that can influence the quality of the educational response that learners receive. In the case of gifted and talented students, this relationship has been extensively tested in national and international studies, and it has even been observed that teachers who are more committed to the concept of inclusion do not always have favourable attitudes towards these students (McCoach and Siegle, 2007; Tirri, Tallent-Runnels, Adams, Yuen and Lau (2002). The aim of this study was to test the relationship between teachers' attitudes towards inclusion and towards students with high abilities and their education. A total of 203 active teachers (56 men and 147 women) from different educational levels, aged between 22 and 74 years, 48.53 (15.5) years, answered two questionnaires: the Questionnaire of teachers' perceptions about inclusion (Cardona, Gómez-Canet \& González-Sánchez, 2000) and Opinions about students with high abilities and their education (Original by Gagné \& Nadeau (1991, and adapted by Tourón, Fernández \& Reyero, 2002). It was found that younger and better-trained teachers show more favourable attitudes towards inclusion. However, these characteristics do not always coincide when we assess their attitudes towards gifted students.
\end{abstract}

Keywords: teacher attitudes; educational inclusion; educational needs; gifted students

\section{INTRODUCCIÓN}

Ya en 1994, la Declaración de Salamanca proclama la inclusión como el medio más eficaz para educar a todos los alumnos en el sistema educativo ordinario y para que éstos accedan a una educación de calidad independientemente de sus características y necesidades individuales (Unesco, 1994). Esto supone que todos los alumnos de una determinada comunidad aprendan juntos, independientemente de sus condiciones personales, sociales 0 culturales, incluidos aquellos que presentan una discapacidad (Reyes, 2010). Existen distintas técnicas que el profesorado puede utilizar en sus clases para dar respuesta a las distintas capacidades y características de los alumnos y conseguir así una inclusión plena (Vicuña, 2013), pero también se detectan muchos obstáculos para el logro de este objetivo. Entre los más importantes se encuentran las actitudes del profesorado. Según la Teoría de la acción razonada (Ajzen, 2012), existe una clara relación entre las creencias y actitudes hacia un colectivo y el comportamiento hacia este.

En la actualidad, se concibe Alta Capacidad Intelectual (de aquí en adelante AACC) como un fenómeno multidimensional, que se manifiesta de distintas formas y en diferentes niveles, según las personas, las circunstancias y el momento que se trate. Según la National Association for Gifted Children (2013; 2020) las personas con AACC son aquellas que demuestran un nivel de aptitud sobresaliente (capacidad excepcional para razonar y aprender) 0 competencia (desempeño documentado o rendimiento que los sitúe en el 10\% superior al grupo normativo) en uno 0 más dominios.

En el Decreto que regula la respuesta educativa a la diversidad del alumnado en la Comunidad Autónoma de Extremadura (Decreto 228/2014, de 14 de octubre), podemos localizar una referencia al Alumnado con Altas Capacidades Intelectuales en el capítulo 1. Disposiciones Generales, en sus Principios de Actuación, el artículo 17.1. donde se expone que, se considera alumnado con necesidades específicas de apoyo educativo por altas capacidades intelectuales, a aquel cuya evaluación psicopedagógica determine que dispone de una capacidad intelectual superior a la media, con evidencia de una elevada productividad, un alto nivel de creatividad y un alto grado de dedicación a las tareas. Un problema que se plantea, es que muchos docentes que se muestran de acuerdo con la filosofía de la inclusión educativa, no visualizan al alumnado de AACC dentro del grupo de alum- 
nos a los que habría que ofrecer una atención especial. Por ejemplo, en la reciente publicación de Van Hove, De Schauwert, Verdugo, Calvo y Amor (2019) sobre la situación de la Inclusión educativa en España, se alude mínimamente al alumnado de AACC y sólo se hace cuando se habla de aspectos "legales". El desconocimiento de las necesidades reales de este colectivo, entre los propios profesionales educativos especialistas, les conduce a considerar que los recursos destinados a atender a este colectivo restarían opciones a los alumnos que "realmente" Io necesitan (McCoach \& Siegle, 2007) dando lugar a un supuesto conflicto de suma cero, cuando lo que deben buscar los defensores de ambos colectivos es una educación personalizada que ayude a cada niño a desarrollar todo su potencial.

Así, atender a los alumnos con AACC, ¿es elitista y reduce los recursos que deben destinarse a otros alumnos con necesidades educativas especiales?. Esta pregunta tendría una doble respuesta. La primera de ellas es que estos alumnos también tienen necesidades educativas, reconocidas por la legislación, a nivel europeo, nacional y de todas las Comunidades autónomas de nuestro país (Gómez Labrador, Gozalo Delgado, León Barco y Mendo Lázaro, 2019). La segunda enlaza con la importancia del aprendizaje personalizado, defendido por numerosos autores como Tourón y Freeman (2017). Este enfoque se adapta al ritmo, intereses, preferencias y necesidades de todo el alumnado, entendiendo que los objetivos, los contenidos, el ritmo y el método de instrucción pueden variar de un alumno a otro. En esta misma línea, Fernández, y Sánchez, (2012) afirman que las necesidades educativas especiales de estos alumnos forman parte de la diversidad, pues ningún estudiante aprende de una misma manera, ni al mismo tiempo, ni las mismas cosas. Esto implica que no podemos darle un tratamiento homogéneo desde el centro educativo a todo el alumnado. Se precisa una respuesta que atienda las particularidades de cada alumno, en lugar de una respuesta común.

Para que la educación inclusiva y transformadora se produzca, tienen que cumplirse una serie de factores. Así, las actitudes del profesorado, que Verdugo, Arias y Jenaro (1994) definen como ideas cargadas de emoción que predisponen a ciertas acciones ante determinados estímulos, cuentan con tres componentes constituyentes: la idea (componente cognitivo), la emoción asociada a dicha idea (componente afectivo) y la predisposición a la acción (componente conductual). Por eso, resulta esencial encontrar formas efectivas para apoyar al profesorado en el desarrollo de su capacidad para enseñar a todos los alumnos (Ainscow, 2007). Otros estudios han explorado factores como los años de experiencia de los docentes, su edad, género o la etapa educativa en la que trabajan. Si nos referimos a la edad, los resultados de los estudios no son concluyentes: algunos no encuentran relaciones claras entre ambas variables. También encontramos trabajos que indican que los docentes con más años de experiencia son los que tienen una actitud más favorable hacia la inclusión (Van Reusen, Shohoy Barker, 2000; Vicuña, 2013).

Numerosos autores coinciden en que las actitudes del profesorado hacia el alumnado de AACC tienen un impacto crucial en la calidad de la educación de los estudiantes y sus logros (Brulles, Saunders y Cohn, 2010; Jacobs y Harvey, 2010; McCoach y Siegle, 2007). Estos alumnos necesitan profesores que entiendan sus necesidades educativas y emocionales, que se muestren cercanos y se preocupen por ellos (Folson, 2009). Se ha encontrado una clara correlación entre la motivación del estudiante y el entusiasmo que presentan los docentes por enseñar a un alumnado de estas características (Matheis, Kronborg, Schmitt, y Preckel, 2018). Sin embargo, gran parte de los docentes carecen de formación y experiencia y, por lo tanto, se guían por creencias basadas en su conocimiento implícito, el cual suele estar basado en mitos, estereotipos y creencias. Kunter y Holzberger (2014) indican que las creencias incorrectas conducen a evaluaciones negativas de estos alumnos que, además, pueden afectar negativamente a las expectativas de los docentes y a su comportamiento y actitud con este alumnado.

\section{OBJETIVOS DE LA INVESTIGACIÓN}

Los objetivos de este trabajo son: 1) comprobar si la edad y los años de experiencia de los docentes influyen sobre sus actitudes hacia la inclusión y hacia las AACC y 2) valorar si aquellos docentes que tienen mejores actitudes hacia la inclusión educativa son los que están mejor predispuestos a atender las necesidades educativas especiales del alumnado con AACC 


\section{MUESTRA Y/O PARTICIPANTES}

En total, participan 203 docentes de la comunidad de Extremadura, utilizando un muestreo por conveniencia. Sus características pueden consultarse en la tabla 1.

Tabla I. Datos descriptivos de los participantes en el estudio.

\begin{tabular}{|c|c|c|c|c|c|}
\hline & & $\mathbf{n}$ & $\%$ & $\overline{\mathbf{x}}$ & $\boldsymbol{\sigma}$ \\
\hline \multirow{2}{*}{ Género } & Hombre & 56 & 27.59 & & \\
\hline & Mujer & 147 & 72.41 & & \\
\hline \multirow[t]{4}{*}{ Edad } & & 203 & 100 & 48.53 & 15.50 \\
\hline & Educación Infantil & 41 & 20.20 & & \\
\hline & Educación Primaria & 46 & 22.66 & & \\
\hline & $\begin{array}{l}\text { Secundaria, Bachillerato o } \\
\text { ambas }\end{array}$ & 87 & 42.86 & & \\
\hline \multirow[t]{4}{*}{ Etapa educativa } & Infantil y Primaria & 14 & 06.90 & & \\
\hline & $\begin{array}{l}\text { Otras combinaciones y/o } \\
\text { Universidad }\end{array}$ & 15 & 07.39 & & \\
\hline & Magisterio & 96 & 47.29 & & \\
\hline & $\begin{array}{l}\text { Grado/licenciatura en otras } \\
\text { temáticas }\end{array}$ & 90 & 44.33 & & \\
\hline \multirow{3}{*}{$\begin{array}{l}\text { Especialidad como } \\
\text { docentes }\end{array}$} & Grado/licenciatura y máster & 6 & .03 & & \\
\hline & Técnico sin titulación & 1 & .01 & & \\
\hline & Doctorado & 10 & .05 & & \\
\hline $\begin{array}{l}\text { Años de } \\
\text { experiencia } \\
\text { docente }\end{array}$ & & 203 & 100 & 22.56 & 14.64 \\
\hline \multirow{3}{*}{$\begin{array}{l}\text { Localización del } \\
\text { centro }\end{array}$} & Rural & 67 & 33.00 & & \\
\hline & Urbano & 136 & 67.00 & & \\
\hline & Centro concertado & 13 & 6.40 & & \\
\hline \multirow{2}{*}{ Carácter del centro } & Centro público & 172 & 84.73 & & \\
\hline & Centro privado & 20 & 9.85 & & \\
\hline
\end{tabular}

n: participantes; \%: porcentaje; $\bar{x}$ : media; $\sigma$ : desviación típica.

Se contactó con los equipos directivos de varios centros educativos de la Comunidad Autónoma de Extremadura y se solicitó que se difundiera la encuesta entre el profesorado. Debido a las restricciones de movilidad asociadas con el COVID-19, las encuestas se respondieron en formato on-line. Los participantes aportaron un consentimiento informado.

\section{METODOLOGÍA Y/O INSTRUMENTOS UTILIZADOS}

Además de solicitar datos sociodemográficos básicos, se administran dos herramientas. La primera, el Cuestionario de percepciones del profesorado acerca de la inclusión (CPPI) (Cardona, Gómez-Canet y GonzálezSánchez, 2000), explora las percepciones y las actitudes hacia la inclusión. La puntuación final se obtiene a partir de la suma de los 12 ítems que conforman el cuestionario, basados otras tantas afirmaciones sobre la educación de los alumnos con necesidades educativas especiales en el aula ordinaria. El grado de acuerdo de los participantes se expresa utilizando una escala tipo Likert de 5 puntos (desde 1 = nada de acuerdo, hasta 5 = muy de acuerdo). Así, la puntuación mínima será de 12 y la máxima de 70. La escala se organiza en torno a tres factores: Bases de la Inclusión (7 ítems), Formación y Recursos materiales (3 ítems) y Apoyos personales (2 ítems). El análisis de la fiabilidad a través de su consistencia interna es .75. La herramienta que se administra es el cuestionario Opiniones sobre los alumnos con altas capacidades y su educación (OAACE) original de Gagné y Nadeau (1991), y adaptada a la población española por Tourón, Fernández y Reyero, 2002), que consta de 34 ítems que recogen 
una serie de afirmaciones referentes a los alumnos con altas capacidades. El formato de respuesta es tipo Likert de 5 puntos (desde $1=$ totalmente en desacuerdo, hasta $5=$ totalmente de acuerdo). Las puntuaciones oscilan entre 34 y 170. Se divide en 6 factores: Necesidades educativas, Opiniones subjetivas, Valor social, Rechazo, Agrupaciones por alta capacidad y Aceleración. Este cuestionario ha sido el más más empleado en el ámbito internacional para evaluar las actitudes de los docentes. La consistencia interna de la escala final es de .69, no todas los factores alcanzan estos valores.

\section{RESULTADOS ALCANZADOS}

La tabla 2 refleja las respuestas de los docentes a las preguntas del cuestionario sociodemográfico. Un $21,67 \%$ del profesorado considera que tiene un nivel de formación bajo o muy bajo, para atender a este alumnado. Casi un $60 \%$ afirma que ha trabajado con alumnos de Altas Capacidades y hay un $40,9 \%$ que nunca ha trabajado con este alumnado. Un $66 \%$ que indica que nunca ha asistido a actividades formativas relacionadas con dicho alumnado, frente a un $34 \%$ que sí lo ha hecho.

Tabla 2. Frecuencias y Porcentajes formación/experiencia con alumnos con Altas Capacidades.

\begin{tabular}{lccc}
\hline & & $\boldsymbol{N}$ & $\boldsymbol{\%}$ \\
\hline & Muy bajo & 5 & 2.46 \\
Nivel de formación y conocimientos para detectar & Bajo & 39 & 19.21 \\
altas capacidades en sus alumnos. & Medio & 107 & 52.71 \\
& Alto & 48 & 23.65 \\
& Muy alto & 5 & 2.46 \\
\hline \multirow{2}{*}{ Ha trabajado con alumnos con altas capacidades } & Sí & 120 & 59.11 \\
& No & 83 & 40.89 \\
\hline Tienen experiencias con personas con altas & Sí & 146 & 71.92 \\
capacidades & No & 57 & 28.08 \\
\hline Asistencia a actividades relacionadas con las altas & Sí & 69 & 34.00 \\
capacidades & No & 134 & 66.00 \\
\hline
\end{tabular}

n: participantes; \%: porcentaje.

En la tabla 3 se recogen los valores obtenidos por los docentes en las puntuaciones de las escalas CPPI y OAACE, así como las relaciones que existen entre éstas y la edad y antigüedad de los docentes. Como se puede observar, el total de la escala CCPPI y la dimensión Bases de la Inclusión muestran correlaciones negativas y significativas con la edad, al igual que Necesidades Educativas y Agrupamiento por Alta capacidad. Es decir, que son los/las docentes más jóvenes quienes presentan mejores actitudes hacia la inclusión, hacia la atención Necesidades de este alumando y a la agrupación en función de la capacidad de los alumnos. La puntuación total en la escala OAACE muestra una correlación positiva con la edad. En el caso de la dimensión de Rechazo, de la escala OAACE, el resultado se debe valorar la inversa, es decir, los docentes mayores sienten menor rechazo por este alumnado y muestran una predisposición global más favorable hacia ellos. 
Tabla 3. Estadísticos descriptivos de los factores de los Cuestionarios de percepciones del profesorado acerca de la inclusión (CPPI) y Opiniones sobre los Alumnos con Altas capacidades y su Educación (OAACE). Correlaciones bivariadas entre estas escalas y la edad, los años experiencia docente

\begin{tabular}{lcccc}
\hline & $\bar{x}$ & $\sigma$ & Edad & $\begin{array}{c}\text { Años de } \\
\text { experiencia }\end{array}$ \\
\hline Escala CPPI & & & & \\
\hline Total CPPI & 42.57 & 8.61 & $\mathbf{- . 2 4 1 * *}$ & $\mathbf{- . 2 2 6 * *}$ \\
Bases de la Inclusión & 27.77 & 5.26 &.$- .170^{*}$ & $-.145^{*}$ \\
Formación y Recursos & 8.24 & 3.05 & -0.06 & -0073 \\
Apoyos Personales & 6.56 & 2.38 & -.018 & .040 \\
\hline Escala OAACE & & & & \\
\hline Total OAACE & 101.85 & 11.37 & $\mathbf{. 2 0 6 * *}$ & $\mathbf{. 1 8 6 * *}$ \\
Necesidades Educativas & 31.03 & 4.57 & $\mathbf{- . 2 1 7 * *}$ & $\mathbf{- . 1 7 2 *}$ \\
Opiniones subjetivas & 23.87 & 6.32 & .009 & .038 \\
Valor social & 11.21 & 2.65 & .056 & .035 \\
Rechazo & 8.37 & 2.50 & $\mathbf{- . 2 4 1 * *}$ & $\mathbf{- . 2 2 6 * *}$ \\
Agrupamiento por alta capacidad & 12.31 & 2.26 & $\mathbf{- . 1 7 0 *}$ & $\mathbf{- . 1 4 5 *}$ \\
Aceleración & 15.05 & 2.26 & -0.06 & -0073 \\
\hline
\end{tabular}

$\overline{\mathrm{x}}$ : media; $\sigma$ : desviación típica.

** La correlación es significativa al nivel 0,01 (bilateral). * La correlación es significante al nivel 0,05 (bilateral).

En cuanto a la relación entre las actitudes hacia la inclusión y hacia la atención al alumnado de AACC (véase Tabla 4) se observa una correlación positiva y significativa entre las dimensiones de la escala CPPI actitudes hacia la inclusión y varias escalas del cuestionario OAACE y su puntuación conjunta: Necesidades Educativas, Opiniones subjetivas, Valoración Social y la puntuación conjunta de la escala. Mientras aquellos participantes que puntúan más alto en Bases e Inclusión y en el conjunto de la escala, obtendrían peores puntuaciones la excepción la dimensión de Agrupación según la capacidad del cuestionario OAACE.

Tabla 4. Correlaciones de Pearson entre los factores del Cuestionario de percepciones del profesorado acerca de la inclusión (CPPI) y el cuestionario Opiniones sobre los Alumnos con Altas capacidades y su Educación (OAACE).

** La correlación es significativa al nivel 0,01 (bilateral). * La correlación es significante al nivel

\begin{tabular}{llllllll}
\hline & $\begin{array}{l}\text { Necesidades } \\
\text { educativas }\end{array}$ & $\begin{array}{l}\text { Opiniones } \\
\text { subjetivas }\end{array}$ & $\begin{array}{l}\text { Valor } \\
\text { social }\end{array}$ & Rechazo & $\begin{array}{l}\text { Agrupación } \\
\text { alta capacidad }\end{array}$ & Aceleración & $\begin{array}{l}\text { OAACE } \\
\text { Total }\end{array}$ \\
\hline Bases e Inclusión & 0.07 & $\mathbf{. 1 9 * *}$ & 0.06 & 0.12 & $-.17^{*}$ & 0.03 & 0.12 \\
Formación y Recursos & -0.1 & -0.05 & 0.09 & 0.04 & -0.13 & -0.12 & -0.09 \\
Apoyos Personales & $\mathbf{. 1 5 *}$ & $\mathbf{. 2 0 * *}$ & $\mathbf{. 2 5 * *}$ & 0.06 & -0.03 & 0.12 & $\mathbf{. 2 2 * *}$ \\
CPPI Total & 0.04 & $\mathbf{. 1 5 *}$ & 0.12 & 0.11 & $\mathbf{- . 1 7 *}$ & -0.01 & 0.09 \\
\hline
\end{tabular}

0,05 (bilateral).

\section{DISCUSIÓN}

Si repasamos el lugar que se ha concedido a la atención a la diversidad, en general y a este colectivo en particular, en las últimas décadas, podemos pensar que el nivel de formación de los docentes en esta temática es bastante mejorable. Ya que, aunque sólo un 21,67\% del profesorado considera que tiene un nivel de formación bajo 0 muy bajo para atender a este alumnado, un $66 \%$ de los participantes indican que nunca ha asistido a actividades formativas relacionadas con dicho alumnado, frente a un 34\% que sí lo ha hecho. A esto debemos añadir 
que un 40,9\% opina que nunca ha trabajado con este alumnado, este resultado sería cuestionable si recordamos que según la estimación de la National Association for Gifted Children (2020), constituyen un 10\% de los alumnos escolarizados.

Según los resultados de la investigación, la edad es una variable que influye en la inclusión y en el empleo de recursos, concretamente, cuanto menor es la edad de los docentes y cuanto menor son sus años de experiencia en el ámbito laboral mejor es su actitud hacia la inclusión. Estos resultados se contradicen con los de Van Reusen et al., (2000) y Vicuña (2013) ya que según sus investigaciones son los docentes de mayor edad los que presentan mejores actitudes hacia la inclusión, como ya hemos visto, otros estudios no observaron relaciones claras entre ambas variables. Sin embargo, nuestros resultados coinciden con los de Balboni y Pedrabissi (2000) consideran que los docentes de mayor edad son los que presentan actitudes más positivas ante el alumnado de AACC.

En cuanto a la relación entre las actitudes hacia la inclusión y hacia la atención al alumnado de AACC, nuestros resultados no han coincido con los de estudios previos (McCoach y Siegle, 2007; Tirri et alt., 2002). Los participantes con mejores actitudes hacia la inclusión también se han mostrado favorables a la atención a estos alumnos en algunas de las dimensiones evaluadas por el cuestionario OAACE. Sin embargo, en los estudios citados esta relación se establecía con especialistas en Educación Especial, mientras que, en nuestro caso, hemos comparado a los docentes en función de la puntuación obtenida en un instrumento. La excepción a esta relación ha sido la dimensión de Agrupación según la capacidad.

Los estudios previos han ofrecido resultados contradictorios. Se ha encontrado que algunos profesores con buenos niveles conocimiento y comprensión de las características de este alumnado y dispuestos a atenderlos en sus clases, mostraban actitudes negativas hacia medidas como la agrupación (en grupos de chicos/as habilidosos/as) y la aceleración. Muchos docentes consideran elitista ofrecer una atención especial a estos alumnos dentro de un aula ordinaria (Winstanley's, 2004) y temen restarle tiempo y atención a otros alumnos. Estudios desarrollados en contextos culturales y geográficos muy dispares, identifican una resistencia ideológica a la diferenciación (Gagné, 1991, Tirri et al., 2002, Curtis', 2005). Los argumentos en contra suelen ser la visión de la equidad por parte de los docentes y la percepción de falta de tiempo para diseñar de estrategias y materiales específicos para este alumnado. Muchos autores explican estas actitudes contrarias a la diferenciación, en función de carencias en la formación de los futuros docentes, que se basa en modelos de enseñanza muy "convencionales" que no facilitan herramientas específicas (Pierce \& Adams, 2009). Por esta razón, perciben la necesidad de adaptarse a las necesidades específicas de los alumnos de AACC como una sobrecarga de trabajo, en un contexto de presión temporal. Estas actitudes negativas pueden deberse a la falta de habilidades para la planificación en el aula (VanTassel-Baska \& Stambaugh, 2005).

Para entender esta medida educativa es necesario profundizar en las necesidades específicas de este alumnado. En España, la edad se sigue viendo como el mejor argumento para establecer las agrupaciones y no se valora la desventaja que supone para los alumnos de AACC, tenerse que adaptar sistemáticamente a objetivos educativos que están por debajo de su potencial y no responden a sus intereses.

Entre las limitaciones de este estudio está el hecho de que se trate de una muestra incidental y no muy numerosa, sobre todo debido a las dificultades encontradas en la situación excepcional del pasado curso 2019/20. Como líneas de futuro sería muy interesante comparar las actitudes de docentes en ejercicio con las de docentes en formación y las de especialistas en Educación especial.

\section{CONCLUSIONES}

La formación del profesorado sigue siendo un aspecto clave. Sin conocer las necesidades concretas de cada colectivo, será difícil conseguir una educación inclusiva de calidad.

La apuesta por la educación personalizada aportaría respuestas más ajustadas a las necesidades de todos/as los alumnos/as. Para ello es necesario buscar estrategias de enseñanza/aprendizaje más flexibles y mejorar las actitudes de los docentes. 


\section{LOS DOCENTES QUE CREEN EN LA INCLUSIÓN EDUCATIVA ¿TIENEN UNA ACTITUD FAVORABLE HACIA EL ALUMNADO DE ALTAS CAPACIDADES?}

Las actitudes de los docentes más jóvenes han sido, en general, más favorables a la Inclusión, aunque los más mayores y experimentados muestran menor rechazo y valoran mejor a los alumnos con AACC.

La medida educativa peor valorada, tanto por los docentes más mayores, como por aquellos que se muestran más favorables a la inclusión ha sido la diferenciación o agrupamiento en función del nivel de capacidad de los alumnos.

\section{REFERENCIAS BIBLIOGRÁFICAS}

Ainscow, M. (2007). From special education to effective schools for all: a review of progress so far. Londres: SAGE.

Aizen, I. (2012). Martin Fishbein's legacy: The reasoned action approach. The ANNALS of the American Academy of Political and Social Science, 640 (1),11-27 doi:10.1177/0002716211423363.

Brulles, D., Saunders, R., \& Cohn, S. J. (2010). Improving performance for gifted education. Journal for the Education of the Gifted, 18(1), 74-86.

Balboni, G. y Pedrabissi, L. (2000). Attitudes of Italian teachers and parents toward school inclusion of students with mental retardation: The role of experience. Education \& Training in Mental Retardation \& Development Disabilities, 35, 148-159.

Cardona, M. C., Gómez-Canet, P. F. y González-Sánchez, M. E. (2000). Cuestionario de Percepciones del Profesor acerca de una Pedagogía Inclusiva. Documento no publicado, Universidad de Alicante.

Chiner E. (2011). Las percepciones y actitudes del profesorado hacia la inclusión del alumnado con necesidades educativas especiales como indicadores del uso de prácticas educativas inclusivas en el aula. (Tesis doctoral, Universidad de Alicante).

Copenhaver R.V. \& Mc Intyre, D.J. (1992). Teacher Perceptions of Gifted Student. Roeper Review. 14(3), 151-154. Curtis', 2005).

Curtis, J. (2005). Preservice teachers' attitudes toward gifted Students and gifted education. (Doctoral Dissertation), Columbia University Teachers College, New York, United States.

España. Decreto 228/2014 de 14 de octubre, por el que se regula la respuesta educativa a la diversidad del alumnado en la Comunidad Autónoma de Extremadura. Diario Oficial de Extremadura, 21 de octubre de 2014, núm. 202, pp. 31554-31588.

Fernández, T. \& Sánchez, T. (2012). Dificultades asociadas a las altas capacidades intelectuales. Sevilla: MAD, S. L. Folsom, C. (2009). Attitudes of Gifted Children. Thousand Oaks, CA: Sage Publications.

Gagné, F. (1991). Brief presentation of Gagné and Nadeau's attitude scale: Opinions about the gifted and their education. Université du Québec à Montréal. Québec, Canada.

Gagné, F. y Nadeau, L. (1991). Opinions about the Gifted and their Education. Université du Québec à Montréal. Québec, Canada.

Geake, J. G. y Gross, M. U. (2008). Teachers' negative affect toward academically gifted Students. Gifted Child Quarterly, 52, 217-231.

Glaubman, R. y Lifshitz, H. (2001). Ultra-orthodox Jewish teachers' self-efficacy and willingness for inclusion of pupils with special needs. European Journal of Special Needs Education, 16, 207-223.

Gomez, C., Gozalo, M., Leon, B. y Mendo, S. (2019). Extremadura, verde, blanca y negra. Avances en altas capacidades. International Journal of Developmental and Educational Psychology INFAD Revista de Psicologia, 1 (2), 65-74.

Guirado, Á. (2015). ¿Qué sabemos de las altas capacidades? Barcelona: GRAÓ.

Jacobs, N., \& Harvey, D. (2010). The extent to which teacher attitudes and expectations predict academic achievement of final year students. Educational Studies, 36(2), 195-206.

Kunter, M., \& Holzberger, D. (2014). Loving teaching: Research on teachers' intrinsic orientations. New York: Routledge. 
Matheis, S., Kronborg, L., Schmitt, M \& Preckel, F. (2018). Threat or challenge? Teacher beliefs about gifted students and their relationship to teacher motivation. Gifted and talented international, 33, 1-26.

McCoach, D. B., \& Siegle, D. (2007). What predicts teachers' attitudes towards the gifted? Gifted Child Quarterly, 51, 246-255.

National Association for Gifted Children -CEC (2013). Teacher preparation standards in gifted and talented education. http://www.nagc.org/sites/default/files/standards/research\%20support\%20for\%202013 \%20stands.pdf

National Association for Gifted Children (8 de mayo de 2020). Que es la dotacion. Washington DC. www.nagc.org.

Pierce, R. y Adams, C. (2009). Attitudes of preservice and experienced teachers toward diverse learners: An expanded abstract. (Doctoral Dissertation), Ball State University, Indiana, United States.

Reyes, M. M. (2010). La formación del profesorado, motor de cambio en la escuela del siglo XXI. Revista de Educación Inclusiva, 3(3), 89-102.

Tirri, K. A., Tallent-Runnels, M. K., Adams, A. (2002). Cross-cultural predictors of teachers' attitude towards gifted education: Finland, Hong Kong, and the United States. Journal for the Education of the Gifted, 26(2), 112131.

Tourón, J., Fernández, R. y Reyero, M. (2002). Actitudes del profesorado hacia la superdotación. Implicaciones para el desarrollo de programas de formación. Faísca, 8, 95-110.

Tourón, J. \& Freeman, J. (2017). APA Handbook on Giftedness and Talent. Washington: American Psychological Association (APA).

UNESCO (1994). Declaración de Salamanca y Marco de acción ante las necesidades educativas especiales. París: UNESCO.

Van Hove,G., De Schauwert, E., Verdugo, M.A. Calvo, I.\& Amor, A. (2019) Inclusive Education: The Way Forward. National Report: Spain. European Commission agreement 2017-3338/001-001.

Van Reusen, A. K. Shoho, A.R. y Barker, K.S. (2000). High school teacher attitudes toward inclusion. The High School Journal, 84 (2), 7-20.

VanTassel-Baska y Stambaugh, T. (2005). Challenges and Possibilities for Serving Gifted Learners in the Regular Classroom. Theory Into Practice 44(3), 211-217.

Verdugo, M. A., Arias, B. y Jenaro, C. (1994). Actitudes hacia las personas con minusvalía. Madrid: Ministerio de Asuntos Sociales, Instituto Nacional de Servicios Sociales.

Vicuña, K. D. (2013). Percepciones y actitudes de profesores de primero a séptimo año de Educación General Básica del Distrito Educativo 4 hacia la inclusión del alumnado con necesidades educativas especiales como indicadores de uno de prácticas educativas inclusivas en el aula (Tesis doctoral, Universidad Casa Grande, Ecuador).

Winstanley, C. (2004). Too Clever by Half: A Fair Deal for Gifted Children. Stoke-on-Trent: Trentham Books.) 
